\title{
CHEMOTHERAPY OF GONORRHEA AND ITS COMPLICATIONS WITH SPECIAL REFERENCE TO THE CAUSE AND PREVENTION OF FAILURES *
}

\author{
By A. H. HARKNESS, M.R.C.S., L.R.C.P.
}

BEFORE mentioning the failures I have experienced with chemotherapy in the treatment of gonorrhœa I should like to stress the importance of this great discovery. It has revolutionised the treatment of this widespread disease and has lessened to almost vanishing point the incidence of complications, regardless of the efficiency of adjuvant treatment. It is more of a specific for gonorrhœa than are salvarsan and its derivatives for syphilis. Before its introduction the treatment of gonorrhœa was execrable and, in most people's hands, the results were not much better than they were before the Act of Parliament which prohibited all but qualified medical practitioners to treat this disease. Before this law was passed chemists dispensed for their patients the various balsam preparations. These lessened the discharge but did not cure the disease. Nevertheless, the urethra and the glands opening into it were not traumatised, and eventually the patient's own resistance cured the infection. Between I9I7 and the introduction of chemotherapy most patients were over-treated. Excessive instrumentation and strenuous massage of anything that could be reached by the index finger prolonged the treatment and precipitated many complications. Posterior irrigation with weak and warm solutions gave excellent results and was then, in my opinion, the most important item in the treatment of gonorrhœea. I have notes on 350 cases of acute arthritis, and not one of these cases had had posterior irrigations before developing this complication. In pre-chemotherapy days, providing one had the full co-operation of the patient, posterior irriga-

* Based on a paper read before the Medical Society for the Study of Venereal Diseases, 25th May, 1940. 
tions efficiently carried out always prevented metastatic complications. Lavage is still indispensable in the few cases where chemotherapy fails, in some of the complications of gonorrhœa, and in many cases of nongonococcal urethritis and prostatitis, but it has now lost its place in the treatment of uncomplicated gonorrhœea, in which there is now nothing to choose between the results obtained with chemotherapy alone and with chemotherapy plus irrigations. The unaided therapeutic efficiency of the sulphonamides is of paramount importance in dealing with large numbers of men. During the last war gonorrhœa was a very serious disease, but in this war there should be few difficulties and it should seldom be necessary for any of the fighting forces to leave their units. In most cases treated with M \& B 693 the gonococcal discharge disappears in 24 hours and it seldom persists for as long as 48 hours. Infections innocently contracted by an adult are of very rare occurrence; still it would be a very wise precaution to segregate the patient during this short period. If the discharge persists it is, of course, necessary to evacuate the patient for investigation by an expert.

My paper is based on 6II cases of gonorrhœa of which 450 were uncomplicated and I6I complicated. In the uncomplicated I have not distinguished between anterior and posterior urethritis as I consider that, even in the early cases, the posterior urethra is always involved. The complicated cases include epididymitis, 52 ; acute prostatitis, 8 ; prostatic abscess, 4 ; acute cowperitis, 8 ; acute arthritis, 54 ; gonococcal ophthalmia, 5 ; iritis, 7 ; metastatic conjunctivitis, I4; periurethral abscess, 2 ; warts, 6 ; pericarditis, I. There are also a further 25 cases of primary ano-rectal gonorrhœa.

I have used all the sulphonamide preparations that have been put on the market in this country and many others. In my opinion M \& B 693 and sulphanilamide are therapeutically the most potent and, judging from the failures I have had, there is little to choose between them. M \& B 693 is more rapid in its action than sulphanilamide and a lower dosage is required to effect a cure. When Lloyd and I originally tested it out I gramme was given three times a day for the first week and this was followed by I gramme twice a day for a further week. I have used smaller and larger doses over longer and shorter 


\section{CHEMOTHERAPY OF GONORRHEA}

periods, but I have come to the conclusion that my original fourteen day course gives the best results. Four grammes of sulphanilamide are given daily for four days and then 3 grammes daily for a fortnight. The intake of fluids is not limited, and all patients are persuaded to drink as much water as possible. In my opinion there is a real danger in restricting fluids. The sulphonamides are medicaments that work through the blood stream and not the urine. M \& B 693 is excreted much more slowly than sulphanilamide and in the urine a high percentage of the drug is frequently present in the acetylated form which tends to crystalise out in the urine. A patient recently under my care was taking M \& B 693, I gramme three times a day. On the seventh day he took no fluids and during the evening developed a typical attack of renal colic. During the attack he passed a small amount of urine which obviously contained blood and on microscopic examination showed a large number of crystals of the acetylated derivative of M \& B 693 . The specimen was sent to Mr. E. J. Baines of Messrs. May \& Baker and he reported that the concentration of free M \& B 693 in the filtered urine was $37 \mathrm{mgm}$. per IOO c.c., and the total M \& B 693 was I54 mgm. per roo c.c. A thorough investigation of the urinary tract was carried out by my colleague Mr. F. J. F. Barrington, but nothing abnormal was found.

I have recently been testing M \& B 760 (Sulphathiazole) in the treatment of gonorrhœea and so far the results have been excellent. It is far less toxic and even more rapid in its action than $M \& B$ 693. At present I am giving 4 grammes daily (I gramme six hourly) for five days only without the aid of adjuvant treatment. I hope to report more detailed results in the near future.

If the urethral discharge persists and gonococci are still present after three or four days of efficient dosage, it is useless to continue with this form of treatment, and I have had no success in changing from one preparation to another. These failures, as will be seen later, are invariably due to drug-fastness. On the other hand, in primary urethral infections due to the Staphylococcus albus it often takes as long as ten days before the urine shows signs of clearing.

If there is no urgency I prefer to wait a fortnight or even longer before carrying out tests for cure, as the 
trauma produced by instrumentation and rectal examinations of a recently infected mucous membrane can easily precipitate a non-gonococcal urethritis. During this observation period normal diet is allowed, and also exercise, but alcohol is still withheld and if the urine, at least a three-hours specimen, remains clear with no threads or at most a few light mucous threads, with a few leucocytes, nothing further is done.

The tests for cure include anterior urethroscopy, smears and cultures after massage of the prostate, vesiculæ seminales and Cowper's glands, and an examination before the first morning micturition, preferably on the morning following urethroscopy. If these tests are negative alcohol is allowed, and if the urine remains clear with no threads, and prostatic smears and cultures are still satisfactory the patient is discharged as cured after a month's observation.

Owing to the high relapse rate reported by some workers the cases in this series were kept under observation for much longer periods, 40 per cent. for a year after cure and the remainder for not less than six months.

The gonococcal fixation reaction on the blood serum was also carried out on each patient and although a large percentage of the results were negative on the completion of treatment, more after M \& B 693 than sulphanilamide, I must admit that I occasionally give a clear bill of health when the result is positive, providing of course that all other tests are negative. Such cases are usually in patients who have had previous attacks of gonorrhœa, the treatment having been inefficient and of many months duration. Large amounts of gonococcal antibody appear in the blood serum, and I believe that these may persist for many years, even though the gonococcal infection has entirely disappeared.

I recently carried out tests on 60 stricture cases attending for dilatations at the outpatients' clinics of my colleagues at St. Peter's Hospital. The patients were between the ages of fifty-five and seventy and the most recent gonococcal infection was of thirty-five years standing. Thirty-three per cent. gave strongly positive reactions, and in no case were gonococci detected in the urinary tract. In one patient with a negative reaction to this test the Wassermann and Kahn reactions were strongly positive; he was suffering from a meatal 


\section{CHEMOTHERAPY OF ĠONORRHAEA}

stricture following a primary chancre. It must be remembered that a negative reaction does not mean that gonococci are not present in the secretions as will be seen in the drug-fast cases which will be described later.

There is clinical and laboratory evidence to show that by using smaller dosage in the initial stages the organisms become resistant to the drug.

MacLean, Rogers and Fleming have proved that pneumococci in an infected animal treated with $\mathrm{M} \& \mathrm{~B}$ 693 readily establish a tolerance or fastness to the drug. This makes it essential, they say, that the initial doses should be large. Four cases in my series who had received early inefficient dosage support the view that in some patients gonococci become drug resistant. In one 4.5 grammes of sulphanilamide had been given over a period of two days and the patient was not referred to me until five days after the completion of this short course (Case I). Another took I tablet of M \& B 693 four times a day for four days followed by 3 tablets a day for eight days (Case 4). This drug fastness does not always occur after an initial low dosage, as I have had many cases who have made uninterrupted recoveries on the institution of larger dosage. Cohn states that strains of gonococci grown from patients whose infections were resistant to sulphonamides often showed no resistance in culture. H. Wezel (I938) found that gonococci from cases which had been resistant to treatment with Diseptal $\mathrm{C}$ would not grow in medium containing $\mathrm{r} \cdot \mathrm{o}$ mgm. per cent. of the same drug. H. Felke (I939), on the other hand, tested I40 strains of gonococci for sulphanilamide resistance in culture, and the results obtained suggested the acquisition of sulphonamide resistance in vivo. Laboratory experiments by Westphal and Carpenter (I940) in which gonococci have been successively subcultured on media containing increasing strengths of a sulphonamide compound, have shown that drug resistance can be acquired in vitro. Swain (I940) states that strains of Streptococcus viridans grown from cases of subacute bacterial endocarditis differed in their susceptibilities to the sulphonamides. Strains in cases who were resistant to treatment were also resistant to the drugs in vitro and strains that were influenced by treatment were susceptible.

The four cases of drug fastness already mentioned had developed the condition before seeing me. There are 
also 2 cases in my series whose resistance to the drug developed whilst under my care and it was due to the fact that, owing to toxic effects, the tablets were not taken regularly.

Two further cases of drug fastness (including Case 6) occurred in patients who were under my care throughout their treatment and there is no evidence of early irregular and inefficient dosage. Both patients took the drug regularly and there were no toxic symptoms, but gonococci persisted in the secretions.

In two of my cases, one treated with M \& B 693 and the other with sulphanilamide, failure was due to the fact that the immediate toxic reactions of the drug were so severe that after only one dose the patients refused to continue with the drug. Both cases were cured with irrigations and Albucid. The results obtained with Albucid combined with posterior irrigations, although not dramatic, are definitely better than they were with the old orthodox treatment.

Fifteen patients in my series had toxic reactions-rash or drug fever-usually between the seventh and twelfth days, which necessitated omitting the drug; but in all of these cases no immediate or late relapses were observed. One patient, not included in this series, suffered from a macular eruption after taking 29 grammes of M \& B 693 . In forty-eight hours the rash had faded and as there were still heavy threads in the urine I repeated the drug. After taking I gramme there was a recurrence of the rash, and no more tablets were taken until he saw me again forty-eight hours later, when I was very alarmed to see a bilateral metastatic conjunctivitis; I insisted on I gramme of sulphapyridine being taken three times a day, and prescribed Io per cent. protargol drops. The eyes were normal within forty-eight hours and I continued with the treatment for seven days. This is the only occasion on which I have repeated the drug so soon after one of these toxic manifestations. I have already mentioned that small and irregular dosage, especially in the early stages, appears to upset the normal defence mechanism of the body, and if the course is repeated full dosage should be prescribed.

The occasional occurrence of a severe and often fatal agranulocytosis following chemotherapy must too be regarded as a failure. In most of the fatal cases recorded 


\section{CHEMOTHERAPY OF GONORRHEA}

the dosage was unnecessarily high. Barnet's case was given 80 grammes of M \& B 693 over a period of twentysix days. Nicol's case was in a man aged sixty suffering from a gonococcal epididymitis and he had in all 68 grammes of M \& B 693 over a period of twenty-one days. It is stated that the discharge ceased and the epididymitis was subsiding after seven days' treatment, and it is not understood why the dosage was not lessened or why it was given for three weeks. If large dosage over a long period is considered necessary it is imperative to carry out blood counts at least once a week. I must admit that I occasionally continue with the drug when the blood picture is not satisfactory, but then it is always in a case with a serious lesion which I am anxious to get well before there is permanent damage. The following case illustrates the point.

A man aged 23 was admitted to St. Charles' Hospital in September, I938, suffering from a purulent gonococcal ophthalmia of the left eye. He had been treated elsewhere for twelve months and I was unable to ascertain

TABLE I

\begin{tabular}{|c|c|c|c|c|}
\hline Date. & & $\begin{array}{l}\text { Total Leucocytes } \\
\text { per c mm. }\end{array}$ & $\begin{array}{l}\text { Polymorphs } \\
\text { per cent. }\end{array}$ & $\begin{array}{l}\text { L.ymphocytes } \\
\text { per cent. }\end{array}$ \\
\hline $\begin{array}{l}\text { September 2Ist, I938 } \\
\text { September 22nd, I938 } \\
\text { September 23rd, I938 } \\
\text { September 24th, I938 } \\
\text { September 26th, I938 } \\
\text { October 7th, I938 : } \\
\text { October I2th, I938 : } \\
\text { November I6th, I938 }\end{array}$ & . & $\begin{array}{r}9,500 \\
7,300 \\
7,200 \\
6,550 \\
8,200 \\
8,300 \\
\text { II,000 } \\
8,600\end{array}$ & $\begin{array}{l}37 \cdot 5 \\
48 \cdot 5 \\
46 \\
42 \cdot 5 \\
48 \\
48 \\
40 \\
51\end{array}$ & $\begin{array}{l}55 \cdot 5 \\
48 \\
49 \\
49 \cdot 5 \\
45 \\
46 \\
54 \\
40\end{array}$ \\
\hline
\end{tabular}

if he had taken sulphanilamide before coming under my care. A complete blood count was carried out before M \& B 693 was prescribed but the result was not reported until two days later when the patient had already taken 6 grammes. There were 4,500 leucocytes per $\mathrm{cmm}$., with a differential count of 35 per cent. polymorphs and 60 per cent. lymphocytes. It was decided to delay chemotherapy until I saw the patient again a few days later. There were then 5,000 leucocytes with 45.5 per cent. polymorphs and 45 per cent. lymphocytes. In view 


\section{BRITISH JOURNAL OF VENEREAL DISEASES}

of the serious condition of the left eye it was decided to continue with chemotherapy and Dr. G. W. Goodhart very kindly arranged to have blood counts carried out daily for as long as it was considered necessary. Table I. gives details.

There were no toxic symptoms and after the first short course of M \& B 693 he took I gramme three times a day for seven days followed by I gramme twice a day for a further week. The course was completed on the $5^{\text {th }}$ October. The eye was normal a few days later.

There were five cases with residual non-gonococcal infections on completion of chemotherapy. One was suffering from urogenital tuberculosis (Case 2); two had urethral strictures (including Case 3); one, chronic cowperitis; and one, prostatitis. The residual prostatitis was due to the treatment the patient had received before seeing me. Whilst gonococci were still present in the discharge, glycerine and ichthyol $2 \frac{1}{2}$ per cent. had been instilled into the posterior urethra twice a week with an Ultzmann metal catheter! One stricture case had a positive gonococcal fixation reaction, but smears and cultures were repeatedly negative for gonococci; six months later the serum still gave a positive result.

Cokkinnis and McElligott, I939, in their series of I,268 male and 2Io female cases of gonorrhœa report that 20 per cent. of the cases subsequently relapsed. This figure does not include the chemotherapy failures and early relapses. In the subsequent correspondence which appeared in the British Medical Journal several workers agreed with these findings. I found it difficult to follow the conclusions of Cokkinnis and McElligott, as in I54 of their cases gonococci were not found at the commencement of chemotherapy although it is stated that all the cases were of proved gonococcal infection. The nonspecific organisms found are not stated. We all know how quickly gonococci disappear after chemotherapy, and one is forced to conclude that most of these I54 cases had non-gonococcal infections. Perhaps this explains the high relapse rate. A urinary infection due to a nongonococcal prostatitis, with the exception of an infection with the Streptococcus facalis, will often clear after a course of M \& B 693, but it is very liable to relapse. Nongonococcal relapse is also likely to follow dilatation of a stricture and this, too, is often due to a latent prostatitis.

$$
218
$$




\section{CHEMOTHERAPY OF GONORRHEA}

In my series there were two immediate non-gonococcal relapses, one occurring seven days and the other fourteen days after I4 day courses of sulphanilamide, and they both followed sexual intercourse. The gonococcal complement fixation reaction was negative in each case. These infections cleared after short courses of posterior irrigations, without the aid of further chemotherapy, and after an observation period tests for cure were entirely satisfactory.

There were four late non-gonococcal relapses, two occurring two months and two six months after cure. No gonococci were found in the discharge. All followed sexual intercourse, the incubation periods varied from five to ten days, and it is possible that they were primary non-gonococcal infections. The gonococcal fixation reaction was weakly positive in one of the patients, and in the other three it was negative.

I have already mentioned the cases of drug fastness which are not controlled in any way by chemotherapy, and gonococci are found in the secretions both during and on the completion of chemotherapy.

Immediate gonococcal relapse occurring up to fourteen days after cessation of chemotherapy is usually due to inefficient dosage or to administration for too short a period. On the resumption of chemotherapy with efficient dosage, cure-will usually be obtained. There were four cases in this series who relapsed after my usual course, on the second, third, fifth and ninth days. The first three were permanently cured after a further course of seven days' duration, and the fourth (Case 5) was found to have gonococci in the recurrent discharge from the left para-urethral gland. M \& B 693 was repeated, but five days later the patient developed an arthralgia and a metastatic conjuntivitis. In future I shall always give local treatment to infections of the para-urethral glands.

I cannot understand the high percentage of late gonococcal relapses reported by some workers, as in my "uncomplicated" series there was only one, and it occurred three months after apparent cure. On carrying out tests for cure the gonococcal complement fixation reaction was strongly positive and urethroscopy revealed a stricture of the bulb due to a previous attack of gonorrhœa. The patient was advised to attend for dilatations, 


\section{BRITISH JOURNAL OF VENEREAL DISEASES}

but was not seen again until there was a recurrence of the discharge. As will be seen later two late gonococcal relapses followed acute infections of Cowper's gland. There were, however, three reinfections occurring four months, eight months and twelve months after discharge. The gonococcal fixation reactions were all negative on completion of treatment and the incubation periods of the reinfections were two, three and four days.

My difficulties and anxieties are always experienced during or immediately after completion of the course of chemotherapy. In the early days I was of the opinion that these drugs were not so effective where there was involvement of the glands which open into the urethra. However, after a thorough investigation of all my failures, I am now convinced that the primary cause of the resistant infectious process in the glands is invariably due to drug fastness. Changing from one preparation to another, plus the usual adjuvant treatment, fails to control these infections. Fever therapy is the ideal treatment for these resistant cases, and in two of them (including Case I) the results were dramatic. I gave Dmelcos vaccine intravenously and the urine was clear with no threads 48 hours after the first injection of $\mathrm{I}$ c.c. The ideal method for administering fever therapy is the Simpson Kettering Hypertherm, but unfortunately it has not been available in London since the war began.

\section{GoNococcal CoMPLications}

Gonococcal Arthritis.-One drug-fast case in the " uncomplicated " series (Case 8) developed a mild synovitis of the right knee and a periostitis of the left os calcis during a course of M \& B 693. A further patient (Case 5) who was admitted with a double epididymitis and an infection of a para-urethral gland subsequently relapsed and during a second course of M \& B 693 developed an arthralgia and a metastatic conjunctivitis. I have already mentioned the patient who developed a metastatic conjunctivitis after taking only 2 tablets of M \& B 69348 hours after the termination of a course of 29 grammes.

In this series there were 54 consecutive cases of gonococcal arthritis. Thirty-six were treated with M \& B 693 and I 8 with sulphanilamide. The former was prescribed 


\section{CHEMOTHERAPY OF GONORRHCA}

in my routine I4 days' course, and in ten of the cases it was continued for a further week. Four cases received 4 grammes a day for the first four days, but I did not continue with this dosage as the toxic symptoms were more marked and it did not appear to give any better results. Four grammes of sulphanilamide were given daily for four days, followed by 3 grammes daily for a further fortnight.

I have always considered that disinfection of the posterior urethra was the most important item in the treatment of metastatic complications, and in this series it was given twice daily as adjuvant treatment. Each of the cases received an intramuscular injection of a mixed gonococcal vaccine every five days. Radiant heat was applied to the affected joints for fifteen minutes each day; if the joints were acutely painful they were immobilised slightly with sandbags only, and there was no splinting, even for short periods.

The results obtained with the orthodox treatment of the past compare very favourably with those now obtained with the addition of chemotherapy, although, in some of the early acute cases and in those who have been running a septicæmic course, rapid cures were sometimes obtained. These drugs are found in the synovial fluid ; in one obstinate case a knee was aspirated and Dr. Goodhart reported that the fluid contained $7.5 \mathrm{mgms}$. of M \& B 693 per Ioo c.c. Infected joints of the ankles and small joints of the feet and periostitis of the os calcis were always troublesome and chemotherapy has not been very helpful in dealing with them.

In view of the many failures I have had in the treatment of this complication when giving sulphonamides by the mouth or by intramuscular injections, it is interesting to record the following case which was reported by A. Cain, R. Cattan and T. Arnous (1938). An excellent result followed injections, into the affected knees, of a solution of sulphanilamide in acetylmethylamine, 0.25 grammes per.c.c. On the first day Io c.c. of synovial fluid were aspirated from the left knee and I c.c. of the solution was injected. On the second day 2 c.c. were injected into the right knee and on the third, seventh, ninth and eleventh days 2 c.c. into the left knee. The injections were well tolerated and the joints, which had previously resisted sulphonamides by the mouth, quickly returned to normal. 


\section{BRITISH JOURNAL OF VENEREAL DISEASES}

Many observers consider that secondary organisms play a very important rôle in metastatic gonorrhœa and perhaps this explains why chemotherapy is not so dramatic in its effects as it is in other complications of gonorrhœa.

Further joints became involved in five cases, one on the fourteenth day, after 35 grammes of M. \& B. 693, and the others on the second, fourth, fifth and ninth days. In the one occurring on the second day the patient was taking sulphanilamide (4 grammes daily), and the others, M. \& B. 693.

In a series of 80 consecutive cases treated by orthodox methods before the introduction of chemotherapy I have previously reported that during treatment further joints became involved in II.25 per cent. of the cases and in this series the percentage is 9.4 . One case developed lesions of keratodermia blenorrhagica on the soles of both feet and a keratosis of the glans penis whilst he was taking M. \& B. 693. Another, after a week of sulphanilamide, developed a large lesion of keratodermia blenorrhagica on the scalp and back and fourteen days later, after three weeks of sulphanilamide, the soles of the feet became similarly affected; one week later there was periostitis of the left tibia. After a week's interval a fourteen-day course of M. \& B. 693 was prescribed and on completion of this course there was an acute exacerbation of the joints involved.

In most of the cases the urethral discharge disappeared in 48 hours and the urine became clear with no threads. One case who had previously had two four-day courses of M. \& B. 693 developed a tolerance to the drug, and even though 35 grammes of M. \& B. 693 were given in fourteen days the urine remained muddy. He was suffering from a gonococcal prostatitis and was eventually cured with irrigations and prostatic massage. Three other cases had a residual non-gonococcal prostatitis, and in one there was a urethral stricture admitting a No. I3 (French) bougie (Case 3 ).

Inflammation of the glands of Littré and inflammation surrounding these glands usually react readily to the sulphonamides. In the eight drug-fast cases there was a marked littritis and peri-littritis, and the condition was not controlled in any way by chemotherapy. In six of the cases local adjuvant treatment was subsequently necessary to destroy the lesions, and in two of them (in- 


\section{CHEMOTHERAPY OF GONORRHEA}

cluding Case I) the lesions had entirely disappeared fourteen days after fever therapy alone. In no case has the pathological processes gone so far as to form a periurethral abscess. One patient, not included in this series, developed a urethral stricture four months after a full course of chemotherapy. He had defaulted before tests for cure were carried out.

There were 5 cases of gonococcal ophthalmia, and the results with chemotherapy and adjuvant treatment were far superior to those obtained in such cases before the introduction of this new remedy. It is interesting to note that a further patient who had previously lost his right eye was admitted with an attack of acute gonorrhœa and a purulent gonococcal conjunctivitis of the socket. The inflammation of the conjunctiva cleared in two days.

Metastatic conjunctivitis and iritis were never serious complications and reacted to the old treatment almost as well as they do now. Suffusion of the conjunctiva is occasionally a toxic manifestation of the sulphonamides, and I have seen it mistaken for a metastatic conjunctivitis.

Pericarditis occurred in a patient who was suffering from gonococcal septicæmia with multiple arthritis, iritis and keratodermia blenorrhagica. The attack of pericarditis developed soon after the introduction of chemotherapy, and Prontosil, I gramme three times a day, was given for three weeks; the result was excellent.

Excellent results were obtained in the cases suffering from acute prostatitis which had developed before the commencement of chemotherapy, and in 90 per cent. of the cases it was unnecessary to carry out any subsequent treatment. There was always marked swelling, either one-sided or a generalised enlargement of the whole organ. Catheterisation is still necessary in dealing with the patients who are suffering from retention of urine. On the other hand, in the cases where this complication develops during or soon after an unsuccessful course of chemotherapy, there is always a one-sided-usually the left in my series-hardness and prominence of the side affected, and it is only slightly enlarged. One patient, not included in this series, was sent in with a diagnosis of prostatic calculi. Further courses of the sulphonamides have no effect. They are most difficult cases to treat 


\section{BRITISH JOURNAL OF VENEREAL DISEASES}

and nothing like it was ever encountered in pre-chemotherapy days. I consider the condition is worthy of the name of a "Sulphonamide Prostatitis." In future I shall always treat these cases with fever therapy (Case I).

In gonocococcal epididymitis there are multiple miliary abscesses and in consequence of the smallness of the lesions the sulphonamides are able to reach them vi $\hat{a}$ the blood-stream. In a series of 52 patients suffering from gonococcal epididymitis and treated by chemotherapy alone there were only two failures. Both patients were suffering from a " sulphonamide prostatitis," and it was necessary to carry out further adjuvant treatment. It is surprising that the microscopic abscesses of a gonococcal epididymitis seldom coalesce and form a fluctuating tumour. I have records of four such cases, but they all occurred before the introduction of chemotherapy; still, I feel that they would not react to this form of treatment until the abscess was drained.

The sulphonamides have little or no action on an abscess of the prostate, Cowper's gland, or a periurethral abscess until the abscess bursts spontaneously or is drained. The results obtained in the treatment of cases suffering from acute gonococcal infections of Cowper's glands are now definitely better than they were in pre-chemotherapy days, although in this series of eight cases there were two late relapses, one three weeks and the other (Case 9) four months after clinical cure. If an abscess has formed it is still necessary to evacuate the pus before the institution of chemotherapy. In three of the cases who had retention of urine, catheterisation was necessary.

Warts (condylomata acuminata) seen in association with gonococcal discharges of the urethra and anus are not influenced in any way by chemotherapy and must receive adjuvant treatment. Two patients developed warts during chemotherapy and in one they were situated in the lumen of the external urinary meatus. I find Trichloracetic acid Ioo per cent. the method of choice in their destruction.

There were no failures in the treatment of 25 cases of primary ano-rectal gonorrhœa, Io treated with sulphanilamide and ${ }_{5} 5$ with M. \& B. 693. Adjuvant treatment was also given. It is interesting to note that in all these cases, including one with a gonorrhœal arthritis, there were negative gonococcal fixation reactions 


\section{CHEMOTHERAPY OF GONORRHEA}

throughout treatment and in the subsequent observation periods.

The following cases illustrate some of the failures of chemotherapy.

Case I.-Chemotherapy failure due to drug fastness; subsequent cure after fever therapy. The past history shows that there was an acute thrombosis of the left testicle nineteen years previously, and it was followed by atrophy of the testicle. No previous attack of gonorrhoa.

A male aged 4I contracted gonorrhœea, 29/7/39. He did not seek advice until ten days had elapsed, and at that time had fever and pain and frequency of micturition. He was given sulphanilamide, 4.5 grammes in all, for two days only, and I first saw him five days after the completion of this short course. He had hypospadias. There was a profuse discharge containing gonococci, and the infection was posterior. The gonococcal fixation reaction was negative, and remained negative throughout treatment. M. \& B. 693 was prescribed, but he was unable to attend more than twice a week for irrigations. The gonococcal discharge became less profuse but persisted, and the urine remained muddy. No toxic symptoms were observed, and M. \& B. 693 was omitted after he had taken 35 grammes - seven days 3 grammes a day, followed by seven days 2 grammes a day. Four days later sulphanilamide was tried; and he received 4 grammes a day for four days followed by 3 grammes a day for a further week. The patient's condition remained unaltered, and gonococci were always found in the secretions.

On thorough investigation the anterior urethra showed advanced involvement of Littré's glands and there were well-marked areas of soft infiltration. The left lobe of the prostate was hard and tender and more prominent than the right. Smears and cultures of material expressed from the gland were positive for gonococci. Even though digital examination of the prostate was carried out as gently as possible the patient had a severe prostatic hæmaturia and subsequently developed an acute epididymitis in the atrophied testicle. Two four-day courses of Uleron were given but it had no effect on the disease. The patient was eventually persuaded to attend twice daily for irrigations but even then improvement was slow, and if he omitted irrigations for one day only the gonococcal discharge quickly reappeared. Even in pre- 
sulphonamide days I have never had a case so resistant to treatment.

I decided to try fever therapy. One c.c. of Dmelcos vaccine given intravenously produced a violent general reaction, and since that date the urine has remained clear with no threads. To be on the safe side two further injections, $\mathrm{I} \frac{1}{2}$ and 2 c.c., were given. Tests for cure were negative and there has been no relapse. It is interesting to note that urethroscopy revealed a normal anterior urethra fourteen days after the first injection of Dmelcos. The well-marked areas of soft infiltration had entirely disappeared without the aid of local treatment.

Case 2.-A residual non-gonococcal urinary infection after chemotherapy due to uro-genital tuberculosis. A man aged 44 contracted gonorrhoea, I $4 / 5 / 38$. The previous history shows that he had had syphilis and gonorrhœa 22 years previously.

On examination there was a profuse discharge containing gonococci and the urine was muddy in the first glass and clear with threads in the second. M. \& B. 693, I gramme three times a day was prescribed, without adjuvant treatment. The discharge disappeared in twenty-four hours and did not subsequently return, but the urine still contained threads and was occasionally hazy, even at the end of fourteen days treatment, when he had had 35 grammes of sulphapyridine. Urethroscopy was negative, but smears after prostatic massage contained a large number of pus cells, and cultures yielded a profuse growth of Staphylococcus albus. Posterior lavage was then given twice a day and prostatic massage once a week and the course of M. \& B. 693 was repeated. The urine still contained threads and was occasionally hazy, even after a month's treatment.

The patient was given a rest from all treatment, and fourteen days later he developed a subacute left epididymitis and a large hydrocele. A small fluctuating area which did not communicate with the hydrocele appeared over the epididymis. This was aspirated, and tubercle bacilli were found in the pus. A twenty-four hours' specimen of urine contained a few tubercle bacilli. Subsequent investigation by my colleague, Mr. F. J. F. Barrington, revealed a left renal tuberculosis. Epididymectomy and a left nephrectomy were performed. The patient made an uninterrupted recovery. An examination was made a 


\section{CHEMOTHERAPY OF GONORRHEA}

few weeks ago and the urine remains clear with no threads and is free from tubercle bacilli.

Case 3.-A residual non-gonococcal urethritis due to a urethral stricture. A man aged 35 was admitted to hospital with an enlarged and swollen ankle, and œdema of the right foot. On admission he was found to be suffering from acute gonorrhœal arthritis of both ankles and the small joints of the right foot. There was also an acute inflammation of the plantar fascia and the gonococcal fixation reaction was strongly positive. The urethral discharge persisted, and although it was free from gonococci after twenty-four hours of M. \& B. 693, gram-positive cocci were found daily in the smears, even after a two weeks' course of M. \& B. 693 the urine remained hazy in both glasses. Marked febrile reactions with temperatures up to ${ }^{102}{ }^{\circ}$ followed the weekly vaccine injections which had a beneficial effect on the arthritis. Urethroscopy revealed a stricture in the bulb admitting a I3 French bougie. The stricture was dilated at weekly intervals and the urine was soon clear with only a few threads in the first glass.

Case 4.-Drug fastness due to early inefficient dosage. A man aged 26 contracted gonorrhœa in August, I939. His doctor prescribed M. \& B. 693, I tablet four times a day for four days, followed by 3 tablets a day for eight days.

When first seen by me two days after the completion of this course there was a profuse urethral discharge containing gonococci, and there were symptoms of an acute posterior urethritis ; the gonococcal fixation reaction was negative. The patient was given M. \& B. 693, I gramme three times a day for a week, and I gramme night and morning for a further week. Gonococci were still present, and the urine remained hazy in both glasses, although there were now no acute symptoms. A thorough investigation revealed a left-sided prostatitis, the left lobe being hard and only slightly enlarged. Posterior irrigations with $\mathrm{I} / 6000$ potassium permanganate were given twice a day. Courses of sulphanilamide and Uleron were also given, but they had no beneficial effect. Irrigations and prostatic massage eventually effected a cure after three months' treatment. The cure would have no doubt been more rapid if one had used protein shock methods as in Case I. The gonococcal fixation test was weakly positive seven months after cure. 
Case 5.-Failure of chemotherapy where there is glandular involvement. A man aged 24 contracted gonorrhœa, I5/8/38. He was admitted under my care six weeks later when he was suffering from a double epididymitis, a periurethral abscess and inflammation of the left paraurethral gland. Gonococci were present in the urethral and para-urethral discharge. The gonococcal fixation test did not become positive until three weeks after admission.

There was apparent cure after 2I days' treatment with M. \& B. 693 (45 grammes). In order to test out the efficacy of chemotherapy where there was involvement of a gland which could be kept under direct observation I did not give any adjuvant treatment to the left para-urethral gland. Nine days later slight redness of the duct of the left para-urethral gland was noted, and on pressure of the gland a small bead of pus containing gonococci was seen to exude from the orifice of the duct. There was no urethral discharge, and the urine was clear with no threads. Twenty-four hours later there were signs and symptoms of an anterior urethritis. M. \& B. 693 was prescribed, but the infection spread to the posterior urethra, and five days later the patient developed an arthralgia and a metastatic conjunctivitis. Irrigations were then prescribed, and I minim of carbolic acid was injected into the left para-urethral gland. A further month's course of irrigations aided by a course of Albucid was necessary to clear up the residual prostatitis. In the first place the urethral infection and the double epididymitis reacted to M. \& B. 693, but when unaided by adjuvant treatment it failed to cure the infection of the left para-urethral gland.

Case 6.-Acute gonorrhoea with drug fastness and glandular involvement which did not react to chemotherapy. No evidence of early inefficient dosage. A fireman, aged 20, contracted acute gonorrhœa, 22/5/39. The infection, when first seen by me three days later, was definitely posterior. The gonococcal fixation test was negative and remained negative throughout the period of observation.

He received courses of M. \& B. 693, sulphanilamide and Albucid, but they had no effect on the disease. The tablets were taken regularly and there is no evidence of early inefficient dosage. There was marked involvement of the glands of Littré, a left-sided prostatitis, and a right epididymitis developed during treatment. The 


\section{CHEMOTHERAPY OF GONORRHEA}

condition was eventually cured three and a half months later with posterior irrigations, prostatic massage and Kollmann's dilatations. Fever therapy would have effected a more rapid cure.

Case 7.- Non-gonococcal relapse following dilatation of a stricture due to a previous attack of gonorrhoea. An attack of acute gonorrhœa was cured after a fourteen days' course of M. \& B. 693 combined with posterior irrigations.

Whilst carrying out tests for cure urethroscopy revealed a stricture of the bulb due to a previous attack of gonorrhœa. After dilating the stricture the urine, which was previously clear with no threads, became muddy in both glasses and cultures of the urine yielded a profuse growth of Bacillus coli. M. \& B. 693, I gramme three times a day, was given for two days and the urine once again became clear with no threads. There was a similar relapse after each subsequent dilatation.

Case 8.-Drug fastness with glandular involvement. Before being referred to me this patient had been treated for I4 days with sulphanilamide in a daily dosage of only 2 grammes.

At the first visit gonococci were found in the urethral discharge and there was a subacute left-sided "sulphonamide" prostatitis. The gonococcal fixation test was negative and remained negative throughout. M. \& B. 693 was prescribed and after ten days' treatment he developed a periostitis of the left os calcis and pain and slight swelling of the right knee. He was eventually cured with posterior irrigations and prostatic massage. $\mathrm{He}$ was a suitable case for fever therapy.

Case 9.-Acute infection of Cowper's gland. Late gonococcal relapse. A man aged 27 contracted acute gonorrhœe 24/4/38 and had had no treatment when he saw me a fortnight later. The history shows that three years previously he had attended Janet and Marion for an attack of acute gonorrhœa which was complicated by an abscess of the left diaphragmatic gland of Cowper. The abscess was incised on two occasions.

On examination there was a profuse urethral discharge containing gonococci; the urine was muddy in both glasses and the gonococcal fixation test was strongly positive. There was a large, hard and painful swelling on the left side of the perineum and on rectal examination it was found to communicate with a similar swelling in 


\section{BRITISH JOURNAL OF VENEREAL DISEASES}

the region of the left diaphragmatic gland of Cowper. Sulphanilamide, 4 grammes daily for four days followed by 3 grammes daily for a further week was prescribed, but in view of severe toxic symptoms it was not continued for a longer period. Posterior irrigations were also given twice daily. The pain disappeared after two days' treatment and a week later the left Cowper region was normal on palpation. Treatment was discontinued on $18 / 6 / 38$ and four months later there was a gonococcal relapse, the patient denying the possibility of reinfection. There was a profuse gonococcal discharge and a painful swelling of the same Cowper region. The previous course of treatment was repeated and in fourteen days the urine was clear with no threads. All tests were negative with the exception of the gonococcal fixation test which remained strongly positive.

Nine months later the patient was reinfected and was seen by me on the day the first symptoms were observed. Gonococci were found in the discharge and the second glass of urine was clear. During this attack there was no involvement of the left -Cowper gland.

\section{Summary OF FAILURES}

Uncomplicated.-There were 8 failures due to drug fastness and 5 apparent failures due to residual nongonococcal infections. In 2 cases, owing to the immediate toxic effects of M. \& B. 693 and sulphanilamide, the cure was completed with Albucid and posterior lavage. There were 4 immediate gonococcal relapses occurring on the 2nd, 3rd, $5^{\text {th }}$ and 9 th days after completion of chemotherapy and before the tests for cure had been carried out. Three were cured after a further 7 days' course; the other was suffering from an infection of a paraurethral gland which was destroyed by local treatment. There was only one late gonococcal relapse in the series. There were two immediate and four late non-gonococcal relapses; they all followed sexual intercourse and were in all probability primary infections due to non-specific organisms.

Complicated.-There was a large percentage of failures in gonococcal arthritis. The sulphonamides have little or no action on a periurethral abscess, an abscess of the prostate, or Cowper's glands until the abscess is drained. 


\section{CHEMOTHERAPY OF GONORRHEA}

Warts are not influenced in any way by chemotherapy. There were two late gonococcal relapses following acute infections of Cowper's gland.

\section{REFERENCES}

Barnett, H. L., Hartmann, A. F., Perley, A. M. \& Ruhoff, M. B. (1939) J. Amer. Med. Ass., 112, 5 I8. Cain, A., Cattan, R. \& Arnous, J. (I938) Bull. Soc. Med. Hop. Paris, Сонn, A. (1938) Amer. J. Syph., 22, I.

54, I733.

Cokninis, A. J. \& McElligott, G. L. M. (1939) Brit. Med. J., ii, Io8o. FELKE, H. (I939) Klin. Wschr., 18, 568.

Maclean, I. H., Rogers, K. B., \& Fleming, A. (I939) Lancet, i, 562. NiCoL, H. \& FreEdMAN, B. (I939) Lancet, ii, 647.

Swain, R. H. A. (1940) Brit. Med. J., i, 722.

Westphal, L., Charles, R. L. \& Carpenter, C. M. (I940) Vener.Dis.

Wezel, H. (I938) Münch. med. Wschr., 85, I897. 